J. Phys. IV France 138 (2006) 251-257

(C) EDP Sciences, Les Ulis

DOI: 10.1051/jp4:2006138029

\title{
Utilisation des matériaux luminescents pour la métrologie des faisceaux intenses UVX d'impulsions ultracourtes
}

\author{
N. Fedorov ${ }^{1}$, V. Nagirnyi ${ }^{2}$, A. Vasil'ev ${ }^{3}$, A. Belsky ${ }^{1}$, B. Carré ${ }^{4}$, E. Feldbach ${ }^{2}$, \\ J. Gaudin ${ }^{5}$, G. Geoffroy ${ }^{5}$, S. Guizard ${ }^{5}$, M. De Grazia ${ }^{4}$, M. Kirm², P. Martin ${ }^{1}$, \\ H. Merdji ${ }^{4}$ et G. Petite ${ }^{5}$ \\ ${ }^{1}$ Laboratoire CELIA, Université de Bordeaux I, 33400 Talence, France \\ ${ }^{2}$ Institute of Physics, University of Tartu, Riia 142, 51014 Tartu, Estonia \\ ${ }^{3}$ Department of Optics and Spectroscopy, Moscow Lomonosov University, \\ 11234 Moscow, Russia \\ ${ }^{4}$ Service des Photons Atomes et Molécules, CEA/Drecam, 91191 Gif-sur-Yvette, France \\ ${ }^{5}$ Laboratoire des Solides Irradiés, CEA/Drecam, CNRS and École Polytechnique, \\ 91128 Palaiseau, France
}

\begin{abstract}
Résumé. Récemment nous avons débuté des séries d'expériences sur l'interaction d'impulsions intenses VUV avec les solides luminescents. Le but principal en est l'étude des mécanismes d'interaction et de transfert d'énergie entre les excitations électroniques dans les solides à large bande interdite. L'application directe de ces études sera le développement de matériaux et de méthodes de métrologie pour les faisceaux UVX intenses. Nous présentons les résultats obtenus pour $\mathrm{CdWO}_{4}$ qui montrent que ce cristal pourra servir comme un scintillateur pour le rayonnement VUV intense.
\end{abstract}

\section{INTRODUCTION}

Le développement des sources intenses de rayonnement UVX d'impulsions ultra courtes pose le problème de la métrologie de ce rayonnement. Actuellement les matériaux luminescents sont largement utilisés pour la métrologie de différents rayonnements ionisants. Il existe des scintillateurs spécialisés pour la détection des rayons $\mathbf{X}$ et $\gamma$, des neutrons et neutrinos. Ces dernières années ont vu l'adaptation avec succès de scintillateurs $X$ existants pour la métrologie des faisceaux $X$ intenses de rayonnement synchrotron [1]. L'utilisation de systèmes similaires pour la métrologie des faisceaux intenses VUV parait très attirante, mais il existe cependant plusieurs contraintes à contourner. La différence principale entre l'utilisation des scintillateurs pour la détection des rayonnements X et VUV est déterminée par la forte différence des profondeurs de pénétration des photons dans les matériaux. Les photons VUV d'énergie inférieure à $1 \mathrm{keV}$ sont absorbés par un solide dans les couches superficielles inférieures à $100 \mathrm{~nm}$. Ainsi une impulsion VUV peut créer une densité importante d'excitations dans cette épaisseur et l'interaction entre ces excitations électroniques peut alors perturber la linéarité de la réponse luminescente. On constate en effet, voir les récentes études [2,3], que sous l'excitation par des impulsions VUV intenses de durée femtoseconde, la réponse est impérativement non-linéaire ce qui pose le problème de métrologie de ces faisceaux par la technique luminescente. Une solution possible, que nous proposons dans cet article, est basée sur la mesure de l'image de faisceaux avec résolution temporelle et la reconstruction de la distribution de l'intensité initiale en utilisant les connaissances sur la réponse de scintillateur. Comme scintillateur modèle pour cette présentation nous avons choisi le cristal de $\mathrm{CdWO}_{4}$. 


\section{MODÈLE DE L'INTÉRACTION DES EXCITONS}

La description de la luminescence dans les conditions où il existe une probabilité de destruction des centres luminescents (de concentration $n(\mathbf{r}, t)$ ) par interaction (caractérisée par la fonction $g(r, t)$ ) avec une autre excitation électronique (de concentration $c(\mathbf{r}, t)$ ), peut être réalisée en utilisant le système d'équations cinétiques suivantes :

$$
\begin{aligned}
& \frac{\partial n(\mathbf{r}, t)}{\partial t}=-\frac{n(\mathbf{r}, t)}{\tau_{r}}-\frac{n(\mathbf{r}, t) c(\mathbf{r}, t)}{\tau_{r}} 4 \pi \int_{0}^{\infty} K(r) g(r, t) r^{2} d r, \\
& \frac{\partial c(\mathbf{r}, t)}{\partial t}=-\frac{c(\mathbf{r}, t)}{\tau_{c}}-\frac{n(\mathbf{r}, t) c(\mathbf{r}, t)}{\tau_{r}} 4 \pi \int_{0}^{\infty} K(r) g(r, t) r^{2} d r, \\
& \frac{\partial g(r, t)}{\partial t}-D \frac{1}{r^{2}} \frac{\partial}{\partial r} r^{2} \frac{\partial g(r, t)}{\partial r}=-\frac{1}{\tau_{r}} K(r) g(r, t), \\
& n(\mathbf{r}, 0)=\eta_{r} N_{0}(\mathbf{r}), \quad c(\mathbf{r}, 0)=\eta_{c} N_{0}(\mathbf{r}), \quad g(r, 0)=1,
\end{aligned}
$$

où $\tau_{r}$ et $\tau_{c}$ sont les durées de vie de chaque type des excitations, $\eta_{r}$ et $\eta_{c}$ sont les rendements quantiques de création des excitations de chaque type par un photon VUV et $\mathrm{N}_{0}(\mathrm{r})$ est la distribution des photons dans l'impulsion incidente. Un coefficient sans dimension $K(r)$ caractérise le type d'interaction entre les excitations ; il dépend de la distance entre les excitations. Dans le cas de l'interaction dipôledipôle $K(r)=\left(R_{d-d} / r\right)^{6}$, où $R_{d-d}$ est le rayon caractéristique pour ce type d'excitation électrique de l'interaction dipôle-dipôle. Enfin $D \equiv D_{r}+D_{c}$ sont les coefficients de diffusion des excitations qui caractérise l'importance du déplacement d'une excitation durant sa durée de vie.

Si nous admettons que dans l'interaction participent les excitations d'un seul type, par exemple les excitons, et que les coefficients de diffusion sont négligeables par rapport aux dimensions de la zone excitée, le système (1) se simplifie en une seule équation :

$$
\frac{\partial n(\mathbf{r}, t)}{\partial t}=-\frac{n(\mathbf{r}, t)}{\tau_{r}}-\frac{n^{2}(\mathbf{r}, t)}{\tau_{r}} \frac{q}{2} R_{d-d}^{3} \sqrt{\frac{\tau_{r}}{t}}
$$

C'est une équation cinétique représentant l'évolution de la concentration des excitons qui sont en interaction dipôle-dipôle. Cette interaction entre deux excitons est illustrée par le schéma de la figure 1.

Deux excitons de duré de vie $\tau_{r}$ localisés dans la bande interdite du cristal à la distance de l'ordre de $R_{d-d}$ sont capable de disparaître avec la création d'un trou dans la bande valence et d'un électron de conduction avec une énergie comparable avec l'énergie d'un exciton. On peut montrer que la solution

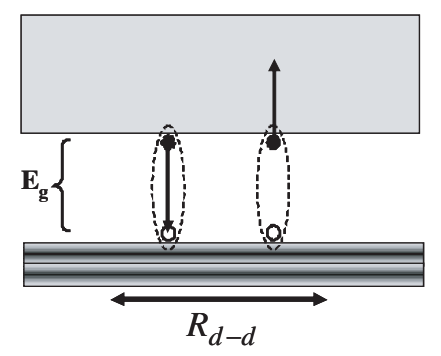

Figure 1. Schéma de l'interaction de deux excitons. 
analytique de l'équation (2) a la forme suivante [4] :

$$
n(\mathbf{r}, t)=\eta_{r} N_{0}(\mathbf{r}) e^{-t / \tau_{r}}\left[1+q \eta_{r} N_{0}(\mathbf{r}) R_{d-d}^{3} \frac{\sqrt{\pi}}{2} \operatorname{erf}\left(\sqrt{t / \tau_{r}}\right)\right]^{-1}, \text { ou } \operatorname{erf}(z) \equiv \frac{2}{\sqrt{\pi}} \int_{0}^{z} e^{-x^{2}} d x
$$

Par l'intégration de cette solution pour un profil gaussien du faisceau VUV: $N_{0}(\rho, z)=$ $I_{0} \frac{\alpha}{\pi \sigma^{2}} e^{-\rho^{2} / \sigma^{2}-\alpha z}$ ou $I_{0}$ est le nombre total de photons dans l'impulsion, $\rho$ - coordonnée $2 \mathrm{D}, \alpha$ - est le coefficient d'absorption, on obtient la relation suivante :

$$
n(t)=\eta_{r} I_{0} e^{-t / \tau_{r}} \frac{-\operatorname{Li}_{2}\left(-\frac{2 \pi^{3 / 2}}{3} \frac{\eta_{r} \alpha I_{0} R_{d-d}^{3}}{\sigma^{2} \pi \sqrt{\pi}} \operatorname{erf}\left(\sqrt{t / \tau_{r}}\right)\right)}{\frac{\sqrt{\pi}}{2} \operatorname{erf}\left(\sqrt{t / \tau_{r}}\right)}
$$

Cette relation (4) exprime la variation en fonction du temps du nombre des excitons émetteurs de photons de luminescence. La figure 2 présente la modification de la courbe de déclin, qui est exponentielle pour une faible intensité d'excitation. Pour ce calcul les conditions similaires aux conditions expérimentales ont été choisies [5]. La variation de la densité d'excitations est réalisée par la variation de la largeur de la distribution gaussienne $(\sigma)$ en fixant les autre paramètres de la relation (4). L'avantage de ce modèle est le nombre très limité de paramètres à faire varier pour la simulation des résultats expérimentaux. Le coefficient d'absorption et le rendement quantique de formation des excitons pour un photon VUV d'énergie donnée sont connus ou peuvent être obtenus à partir d'autres expériences ; le temps de vie radiative $\tau_{r}$ peut aussi être mesuré indépendamment.

L'augmentation de la densité des excitons influe essentiellement sur la partie initiale de la courbe de déclin; l'accélération du déclin est plus forte pour les plus grandes densités (courbes 2 à 4). La surface entre chacune de ces courbes est la courbe 1 est proportionnelle aux nombre des excitons détruits par l'interaction. Le déclin redevient exponentiel avec le temps caractéristique $\tau_{r}$ après un temps $t$ tel que $\mathrm{t} \sim 0.5 \tau_{r}$.

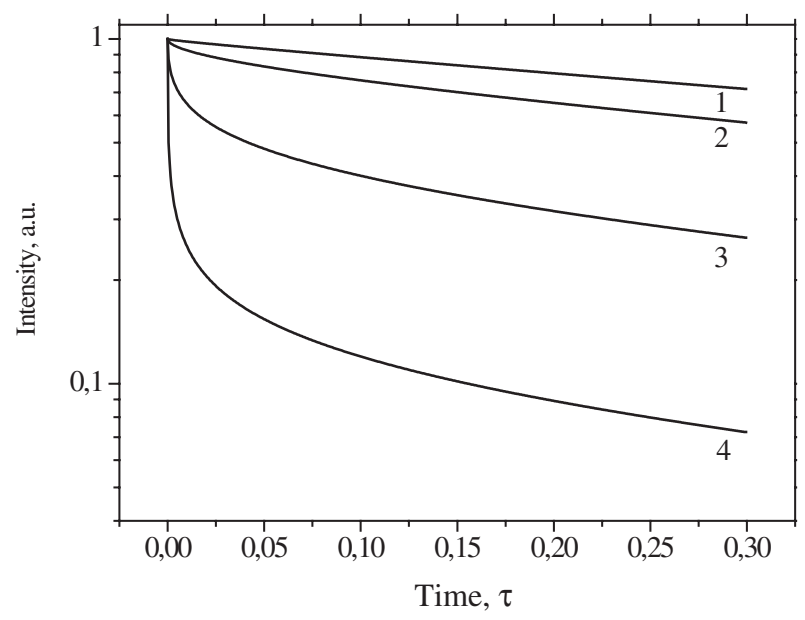

Figure 2. Calcul de la courbe de déclin en utilisant la formule (4). La largeur de la distribution gaussienne et la densité d'excitations au maximum de cette distribution pour chaque courbe sont respectivement : $1-150 \mu \mathrm{m}$ et $10^{13}$ photons $/ \mathrm{cm}^{2} ; 2-50 \mu \mathrm{m}$ et $10^{14}$ photons $/ \mathrm{cm}^{2} ; 3-15 \mu \mathrm{m}$ et $10^{15}$ photons $/ \mathrm{cm}^{2} ; 4-5 \mu \mathrm{m}$ et $10^{16}$ photons $/ \mathrm{cm}^{2}$. 


\section{SIMULATION DE LA RÉPONSE LUMINESCENTE DE CdWO}

Nous avons appliqué le modèle proposé dans la section précédente au cas de $\mathrm{CdWO}_{4}$ pour en déterminer le rayon d'interaction dipôle-dipôle. Le choix de ce cristal pour les études de phénomènes faisant intervenir de fortes densités d'excitations est justifié par le fait que, premièrement $\mathrm{CdWO}_{4}$ est connu pour sa forte résistance aux radiations [6], donc on peut ignorer les effets produits par les défauts. Deuxièmement les excitons dans $\mathrm{CdWO}_{4}$ sont peut mobiles, en effet ce sont des excitons de type moléculaire, localisés sur un oxianion $\left(\mathrm{WO}_{4}^{2-}\right)$ [7], donc leur diffusion est très faible. Enfin l'émission des excitons est intense à la température ambiante et il n'y a pas d'autre type d'excitation électronique susceptible d'être en compétition avec les excitons. Les expériences ont été réalisées au CEA Saclay (DRECAM) en utilisant des impulsions micro-focalisées d'harmoniques d'ordre élevé [5].

La courbe du déclin de la luminescence de $\mathrm{CdWO}_{4}$ est présentée sur la figure 3 et a été mesurée pour un cristal placé au foyer du miroir parabolique focalisant le rayonnement VUV incident sur
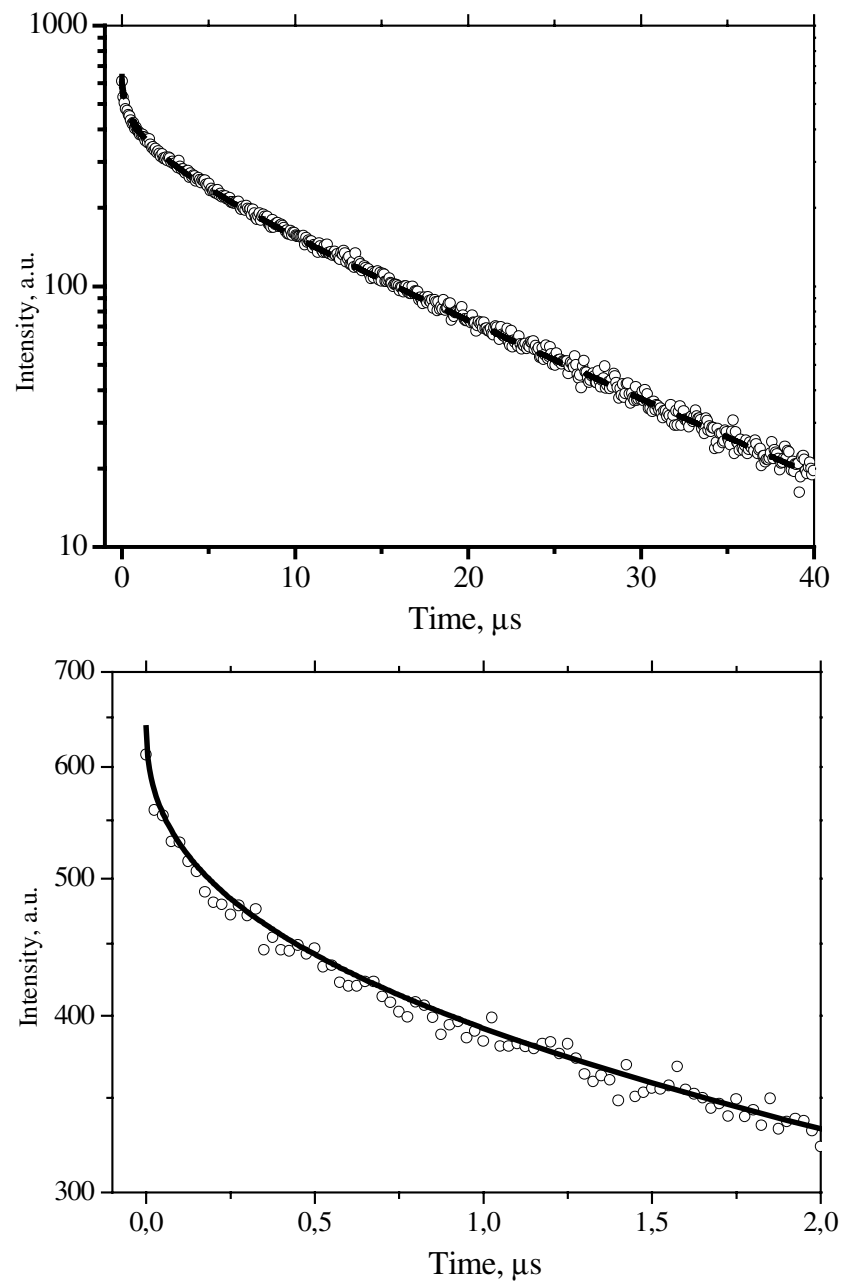

Figure 3. Courbe du déclin expérimental de $\mathrm{CdWO}_{4}$ (ronds) et courbe de simulation (ligne noire). La courbe totale est présentée à gauche et la partie initiale du déclin à droite. 
l'échantillon [5]. Cette courbe montre clairement un comportement correspondant à des effets de l'interaction entre excitons. Pour la simulation nous avons utilisé la formule (4) obtenue pour un faisceau de profil gaussien ; nous avons en effet montré [5] que tel était le cas pour un faisceau observé au foyer. Le nombre total de photon $I_{0}$ dans une impulsion est estimé à $2 * 10^{8}$ photons et nous avons mesuré $\sigma=15.5 \mu \mathrm{m}$ (pour un faisceau à $800 \mathrm{~nm}$ supposé de même profil que le faisceau VUV). Les autres paramètres du solides utilisés pour la simulation sont les suivants : $\alpha=$ $5 * 105 \mathrm{~cm}^{-1}$ et $\tau=15 \mu$ s. Dans ce cas, il ne reste plus qu'un seul paramètre, $R_{d-d}$, à ajuster. Le meilleur accord est obtenu pour une valeur du rayon de l'interaction dipôle-dipôle des excitons dans le cristal de $\mathrm{CdWO}_{4}$ égale à $R_{d-d}=4.3+/-0.4 \mathrm{~nm}$. Cette valeur et tout à fait réaliste pour ce type d'interaction.

On constate donc que le modèle choisi décrit bien les phénomènes produits par un faisceau VUV intense dans le cristal de $\mathrm{CdWO}_{4}$. Ce résultat confirme d'une part l'estimation sur le profil du faisceau VUV et d'autre part les hypothèses effectuées sur la faible mobilité des excitons dans $\mathrm{CdWO}_{4}$ et sur la faible contribution des défauts et autres pièges des excitations électroniques sur le processus de relaxation. Ces propriétés font de $\mathrm{CdWO}_{4}$ un bon candidat pour un scintillateur VUV-X.

\section{MÉTROLOGIE RÉSOLUE EN TEMPS}

L'imagerie du profil d'un faisceau de rayonnement ionisant à l'aide de scintillateurs est basée sur une hypothèse de linéarité de la réponse de ce type de détecteur. Cependant la proportionnalité du rendement de luminescence avec une augmentation de l'intensité d'excitation est limitée. A partir de $10^{13} \mathrm{photon} / \mathrm{cm}^{2}$ environ, il y a un risque que le profil d'une impulsion VUV ne puisse pas être mesuré correctement. Nous avons montré que pour certains scintillateurs, en particulier $\mathrm{CdWO}_{4}$, malgré l'apparition d'une réponse non linéaire à haute intensité d'excitation, cette intensité peut tout de même être reconstruite ; cette reconstruction demande alors la mesure résolue en temps de la réponse de ce scintillateur.

L'illustration de ce procédé est présentée par la figure 4. Supposons qu'un faisceau VUV irradiant un scintillateur génère une tâche de luminescence comme montrée sur la fig.4(a). Pour chaque unité de surface de la tâche la forme du déclin de luminescence possède une allure particulière qui est déterminée par le type de scintillateur et la densité d'excitations en ce point. Ainsi pour un scintillateur de type $\mathrm{CdWO}_{4}$ nous savons alors comment reconstruire à partir de cette courbe le nombre de photons incidents sur cette unité de surface. Techniquement on peut réaliser cette mesure en utilisant une caméra à balayage de fente par exemple ; la précision de la mesure est contrôlée par la résolution temporelle de détection, plus l'intensité incidente est forte et plus l'accélération du déclin les premiers instants est prononcée.

Cependant, il faut signaler que cette technique n'est pas valable que pour une énergie d'impulsion inférieure au seuil de l'ablation du cristal. Car, si la densité d'excitations franchit ce seuil une partie importante de l'énergie absorbée est transformée en déplacement des atomes. L'ablation est accompagnée de la création d'électron chauds qui sont susceptibles de pénétrer dans le cristal dans la direction opposée à la zone ablatée en excitant la luminescence de façon « normale » à cause du fait que la densité d'excitations dans cette profondeur est alors relativement faible.

\section{CONCLUSION}

Dans le cadre d'études expérimentales sur l'interaction d'impulsions intenses VUV avec les solides luminescents dont le but est de mieux comprendre les mécanismes d'interaction et de transfert d'énergie entre les excitations électroniques, nous avons montré qu'il était possible d'obtenir des informations microscopiques telles que le rayon d'interaction dipôle-dipôle. Le cas du cristal de $\mathrm{CdWO}_{4}$ permet ici 

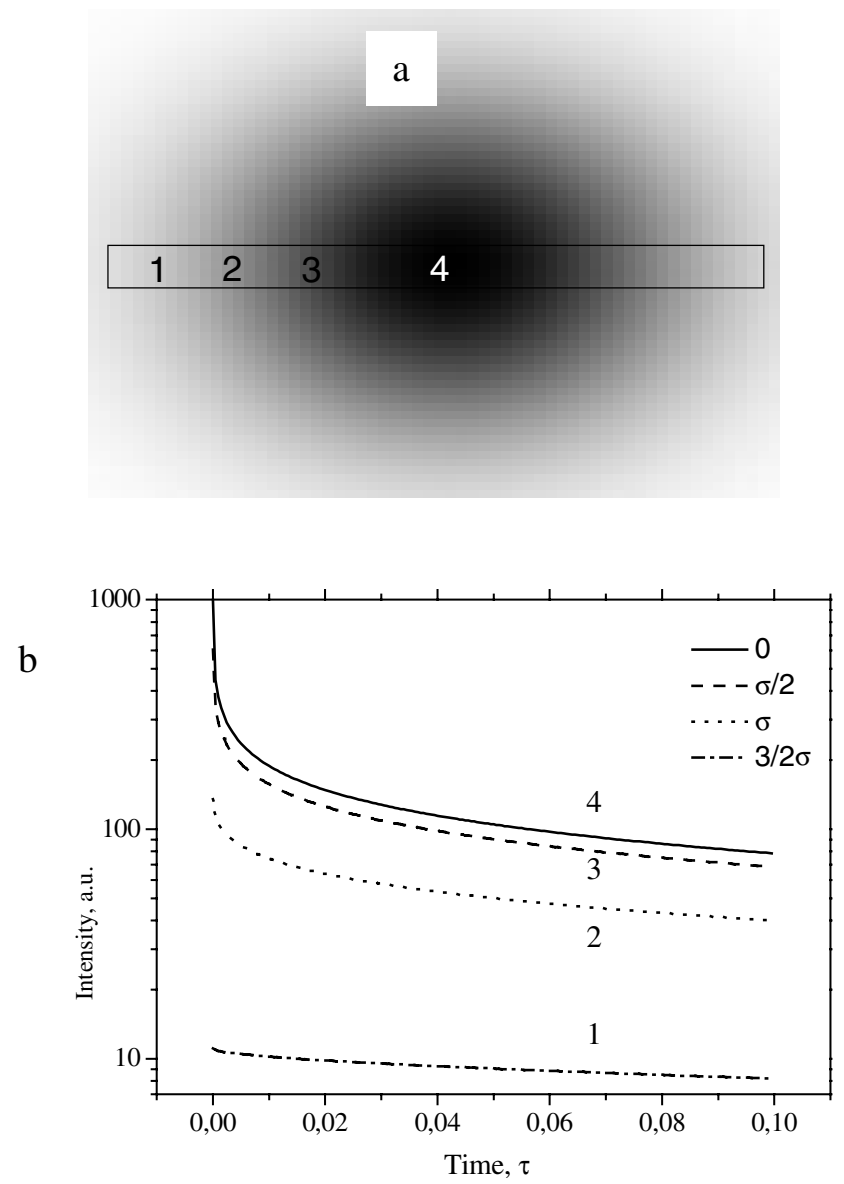

Figure 4. Image de la tâche d'un faisceau de profil gaussien (a) et courbes de déclin de la luminescence de $\mathrm{CdWO}_{4}$ (b) calculées pour différents points de la section horizontale de cette tâche.

une application directe de ces études au développement de méthodes de métrologie pour les faisceaux UVX intenses. Les résultats présentés ici montrent que ce cristal pourra servir comme un scintillateur pour le rayonnement VUV intense.

\section{Remerciements}

Cette étude a été en partie financée par le «MarieCurie Research Training Networks XTRA » (MRTN-CT2003-505138) et le Initiative of Infrastructure LASERLAB-EUROPE (RII3-CT-2003-506350). Nous remercions vivement le personnel du groupe PLFA(CEA/DRECAM) pour son savoir faire, son efficacité et son amabilité de tous les jours.

\section{Références}

[1] Koch, C. Raven, P. Spanne, A. Snigerev, J. Opt. Soc.Am. A15 (1998) 1940.

[2] P. Martin, A. N. Belsky, E. Constant, E. Mével and F. Salin, IEEE Transactions. Nuclear Science 48 (2001) 1137-1142. 
[3] M. Kirm, V. Babin, E. Feldbach, V. Nagirnyi, S. Vielhauer, B. Carré, S. Guizard, M. De Grazia, H. Merdji, A. Belsky, N. Feodorov and P. Martin, Physica Status Solidi (à paraitre).

[4] A.N. Vasil'ev (à publier).

[5] Belsky, N. Fedorov, P. Martin, S. Guizard, G. Geoffroy, J. Gaudin, G. Petite, M. De Grazia, H. Merdji, B. Carré, M. Kirm, V. Nagirnyi, E. Feldbach, A. Vasil'ev, « Interaction d'impulsions VUV intenses avec les solides luminescents », cette issue.

[6] Masaaki Kobayashi, Mitsuru Ishiib, Yoshiyuki Usukic and Hiroshi Yahagi, NIM A, 349 (1994) 407-411.

[7] V. Nagirnyi, M. Kirm, A. Kotlov, A. Lushchik, L. Jönsson, J. Lumin. 102-103C (2003) 597. 